\title{
THE SPECTRAL ERYTHEMIC REACTION OF THE UNTANNED HUMAN SKIN TO ULTRA-VIOLET RADIATION
}

\author{
By W. W. Coblentz, R. Stair, and J. M. Hogue
}

\section{ABSTRACT}

The spectral erythemic reaction of three persons exposed to equal amounts of homogeneous ultra-violet radiation, of various wave lengths, is described. The source of radiation was a standard vertical quartz mercury vapor lamp which has a series of intense conveniently spaced emission lines, extending from 248 to $365 \mathrm{~m} \mu$. These emission lines were separated by passage through the spectrometer exit slit, the jaws of which were dusted with anthracene, , which, by fluorescence, indicated the accuracy of the isolation of the line under investigation.

The erythemal reaction and the radiometric measurements were made on the spectral line directly back of the exit slit. In the region of $250 \mathrm{~m} \mu$ the spectral erythemic reaction was supplemented by exposures to the heterogeneous radiation from a mercury vapor lamp having a high selective emission in the resonance line at $253.7 \mathrm{~m} \mu$. Data, are given on the reciprocity of intensity and time of exposure, and also on the relative spectral erythemic reaction in the range of wave lengths extending from 245 to $315 \mathrm{~m} \mu$.

\section{CONTENTS}

I. Introduction

II. Experimental procedure

III. The reciprocity law

IV. The spectral erythemic reaction

V. Conclusion.

VI. Bibliography

\section{INTRODUCTION}

In connection with an investigation, now in progress, of methous of measuring the dosage of ultra-violet radiation used for healing purposes, the need has arisen for exact numerical data on the spectral erythemic reaction of the untanned human skin to equal amounts of ultra-violet radiation of various wave lengths. Manifestly the amount of ultra-violet radiation that can be applied, without producing a burn, depends upon the tolerance of the skin. This tolerance is widely different for different persons, and widely different even for the same person, depending upon the amount of moisture on the skin.

Hence, the erythemic response is commonly used as an indicator of the amount of ultra-violet radiation that can be applied at any one time. Furthermore, in view of the limitations imposed by skin tolerance, it is highly probable that any unit of dosage, or any inanimate dosage meter that may be adopted will have to take this physiological effect into consideration. This is a safe and logical procedure, even though the spectral erythemic reaction may not be closely related with the spectral reaction in curing disease; for example, rickets.

Hence, in an investigation, now in progress, of the amount of ultraviolet, useful for healing purposes, that is emitted by various sources, 
the radiometric measurements are supplemented by observations of the time and intensity required to produce a minimum perceptible erythema, using measured amounts of ultra-violet from the unknown heterogeneous source of radiation undergoing tests and from the standard source of homogeneous radiation of maximum erythemogenic effectiveness, which, for practical purposes, is the emission line of mercury at $296.7 \mathrm{~m} \mu(1){ }^{1}$

Previous determinations of the erythemic response of the human skin when exposed to ultra-violet radiation of different wave lengths were made by Hausser and Vahle (3) and more recently by Luckiesh (4) and his collaborators. In their determination of the energy required to produce a minimum perceptible erythema, Hausser and Vahle exposed the arm to single emission lines, which passed through a slit (3 cm long) in a cardboard attached to the arm. Based upon their preliminary observations of the approximate magnitude of the erythemal dose for each wave length, by making the exposure through the whole length of the slit, then through two-thirds and finally through one-third of the length, for different time intervals (three exposures with each line), the exposure time for producing a distinctlv perceptible erythema was determined.

Instead of using isolated spectral lines, Luckiesh (4) and his collaborators exposed small areas of the subject's back to the total radiation from a quartz mercury arc lamp, shining through filters that shut off successively increasing wave lengths of ultra-violet radiation, and noting the dosage necessary to produce a minimum perceptible erythema. For this purpose the time was varied so that the shortest exposure gave no visible results, and the longest exposure produced a definite erythema, as observed after a lapse of approximately 24 hours.

\section{EXPERIMENTAL PROCEDURE}

In the present investigation spectral lines (isolated by means of a large quartz monochromator (5) having plano-convex lenses $6 \mathrm{~cm}$ diameter, $18 \mathrm{~cm}$ focal length) were used as stimuli, and the time of exposure to produce a minimum perceptible erythema (one that lasted less than 24 hours) was determined. As found by others, the difficulty lies in defining the minimum perceptible erythema, especially the highly transitory erythema produced by wave lengths less than $270 \mathrm{~m} \mu$ which, for a weak dosage, increases to a maximum redness and then disappears in the course of 2 to 5 hours. In contrast, the erythema produced by the more deeply penetrating radiation, of wave lengths 302 and $313 \mathrm{~m} \mu$, does not begin to be visible until about 4 or 5 hours after exposure. Form this it can readily be seen that on exposure to heterogeneous ultra-violet radiation the erythemal effect of the shortest rays may be waning by the time the erythema, caused by the longer ultra-violet rays, is beginning to be perceptible.

The source of ultra-violet radiation used in the present work was a vertical quartz mercury vapor lamp, operated on a constant voltage (65 volts, 4 amperes in the burner), under which condition the intensities of the spectral lines, measured at different times with a linear thermopile (6) calibrated in absolute value, (7) were constant to 5 per cent.

\footnotetext{
1 Figures in parentheses, here and throughout the text, indicate references given in the bibliography at
} the end of this paper. 
The exit slit (of metal $0.1 \mathrm{~mm}$ in thickness, with beveled edges turned away from the incident light) (8), was mounted upon a movable support which, after focusing upon the desired spectral line, was securely attached to the spectrometer arm.

To facilitate setting on a spectral line the slit was given a thin coat of turpentine upon which was dusted a thin deposit of anthracene, which was then rubbed smooth. Starting with the slit closed, this method of applying the fluorescent material produces a sharp knifeedge which, on opening the slit ( $0.3 \mathrm{~mm}$ wide, $10 \mathrm{~mm}$ long), permits an accurate isolation of closely adjoining emission lines.

The arm of the subject, to be exposed to ultra-violet radiation, rested upon a movable support directly back of, and within less than $0.3 \mathrm{~mm}$ from the front surface of the exit slit. The difference in focus of the radiation incident upon the exit slit, and incident upon the skin directly back of the slit, was therefore negligible. By moving the arm support laterally, different parts of the arm (usually at intervals of 4 to $6 \mathrm{~mm}$ ) could be exposed to radiation. Tests were made on the inner forearm and on the inside upper arm. The three subjects examined were of the white race, dark haired, and of average skin pigmentation.

In practice the exit slit was adjusted to transmit a given emission line (for example, $297 \mathrm{~m} \mu$ ) and a series of irradiation exposures was made, ranging from 1.5, 1.75, 2.0, 2.25, 2.50, and 3.0 minutes. From such a series of exposures the time for producing a minimum perceptible erythema $(1.75,2.0$, and 2.5 minutes respectively, for the three observers, in the winter time when the skin was dry) was easily established. In the summer time, when the skin was moist and the time of exposure for producing a minimum perceptible erythema was only about one-half to two-thirds as long, the intervals between the successive exposures was reduced to 10 seconds instead of 15 seconds formerly used.

When making irradiation tests in the region of $254 \mathrm{~m} \mu$, where the observations of the erythemic reaction are in disagreement with previous experimenters, check exposures were made on the $297 \mathrm{~m} \mu$ line alongside with the exposures to the emission line (for example, $248 \mathrm{~m} \mu$ ) under observation. Since the minimum perceptible erythema for the $297 \mathrm{~m} \mu$ line was produced in the usual time (1.75 minutes; 2 minutes produced an erythema lasting 2 days for W. W. C) it was assumed that this disagreement is real. However, it was realized, at the time (2) that the estimation of a minimum perceptible erythema, which is so transitory as that produced by wave lengths less than $270 \mathrm{~m} \mu$, is subject to an appreciable error, resulting from overexposure. Attention was called to this fact in the preliminary paper (2) on this subject.

In the meantime, the demand having arisen for more accurate data on the spectral erythemic response in the region of $250 \mathrm{~m} \mu$, tests were made with a so-called "cold quartz" mercury vapor lamp (Geissler tube discharge in a quartz tube) in which 95 to 98 per cent of the total ultra-violet erythemogenic radiation of wave lengths less than and including the $313 \mathrm{~m} \mu$ line is contained in the resonance line at $253.7 \mathrm{~m} \mu$.

Using the heterogeneous radiation from such a lamp, it is possible to expose wider areas than 0.3 to $0.4 \mathrm{~mm}$ (which was the maximum 
width in the spectrally isolated homogeneous radiation) and to note the appearance of a faint reddening of the skin that would continue to be visible for 5 to 10 hours, as probably would be done by a physician, using ultra-violet radiation for therapeutic purposes.

\section{THE RECIPROCITY LAW}

According to Luckiesh (9) the reciprocity law holds for biological effectiveness over a wide range of exposures. In the production of a minimum perceptible erythema the reciprocity law was found to hold satisfactorily over the entire range of three hours investigated.

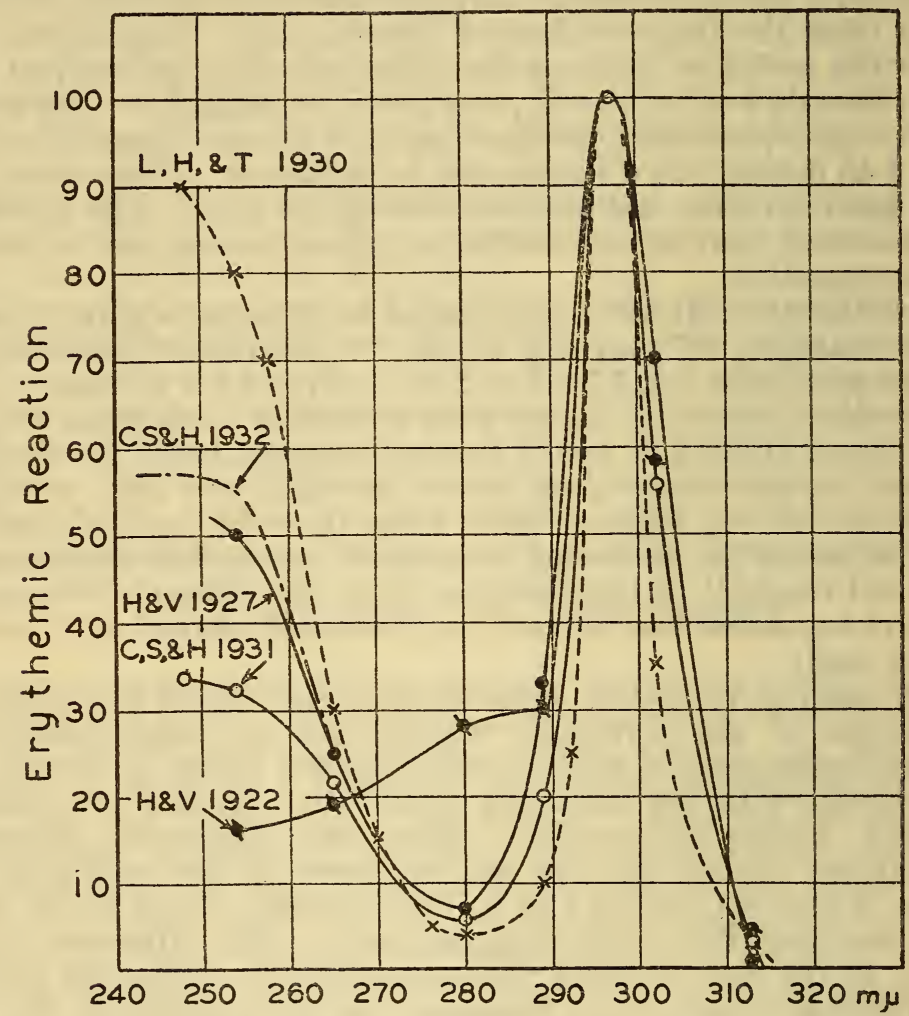

FIGURE 1.-Relative spectral erythemic reaction of the human skin to equal amounts of radiant energy at various wave lengths; $H$. and V., Hausser and Vahle; L. H. and T., Luckiesh, Holladay and Taylor; C. S. and H., Coblentz, Stair, and Hogue

In the present experiments the intensity of the emission line at $297 \mathrm{~m} \mu$ was reduced by one-half, and by one-fourth, by means of glass filters, and the corresponding time of exposure was doubled and quadrupled. Within the experimental errors involved in making the test, the erythema produced by using one-fourth of the unit intensity and exposing for four times the unit time, was not appreciably different from that produced by exposing the subject to unit intensity (430 microwatts per $\mathrm{cm}^{2}$ ) for the unit time (2 minutes for R. S.) required to produce a minimum perceptible erythema. 
From the foregoing tests, it appears that the reciprocal relation of ntensity and time of exposure (the lower the intensity the longer the exposure, and vice versa) is correct for the intensities and corresponding times of exposure that will be encountered in ultra-violet radiation therapy.

\section{THE SPECTRAL ERYTHEMIC REACTION}

As illustrated in Figure 1, the wave length range of the erythemogenic rays begins at about $315 \mathrm{~m} \mu$, and extends to an undetermined wave length shorter than $240 \mathrm{~m} \mu$. The erythemic response curve rises steeply to a maximum at $297 \mathrm{~m} \mu$, descends less steeply to a minimum at about $280 \mathrm{~m} \mu$, then rises to a less intense maximum in the region of $250 \mathrm{~m} \mu$. Considering the difficulties, these magnitudes are in good agreement throughout. However, as already explained, the disagreement of the spectral response curve in the region of 250 $\mathrm{m} \mu$ arises in part from the method of defining the minimum perceptible erythema, which is very transitory for short-wave lengths. This appears to be of minor importance in view of the increased practice of covering artificial light sources with a glass that intercepts ultraviolet radiation of wave lengths less than $280 \mathrm{~m} \mu$, thus reducing the erythemogenic range, of wave lengths to agree more nearly with that of the sun.

From direct experiments on the production of a minimum perceptible erythema using measured amounts of heterogeneous ultra-violet radiation from various sources, including $(a)$ the so-called "cold quartz" lamp (low vapor pressure mercury arc, in which practically all the erythemogenic radiation is contained in the emission line at $253.7 \mathrm{~m} \mu)$, (b) the high-amperage, high vapor-pressure, quartz mercury-arc lamp, and (c) the sun, it is found that the spectral erythemic response, for wave lengths less than $280 \mathrm{~m} \mu$, is represented by the curve (dots and dashes) marked "C. S. \& H. 1932" depicted in Figure 1. In the region of $250 \mathrm{~m} \mu$, this curve is slightly higher than the average value of the various observers.

In their investigations now in progress, involving the determination of the rubescence curve, which is the product of the spectral energy distribution of the source and the spectral erythemic response curve of the skin (analogous to the luminosity curve, which is the product of the spectral energy curve of the source and the visibility of the eye) the writers have adopted, as standard, the spectral erythemic response data, given in Table 1 , in which the wave length of maximum erythemic reaction is taken at the emission line of mercury at $296.7 \mathrm{~m} \mu(2967 \mathrm{~A})$. Excepting at $254 \mathrm{~m} \mu$ these data are practically the average of the various observers. 
TABLE 1.-The relative spectral erythemic reaction of the untanned human skin to equal amounts of radiant energy, averaged (excepting at $254 \mathrm{m \mu}$ ) from the measurements of Hausser and Vahle (spectrally homogeneous radiation 1922 and 1927), of Luckiesh, Holladay, and Taylor (filter method, 1930) and of Coblentz, Stair, and Hogue, using (in 1931) homogeneous radiation, and (in 1932) heterogeneous radiation from a mercury vapor lamp, having 98 per cent of the erythemogenic radiation in the $254 \mathrm{m \mu}$ line

\begin{tabular}{|c|r|}
\hline $\begin{array}{r}\text { Wave } \\
\text { length }\end{array}$ & $\begin{array}{c}\text { Erythemic } \\
\text { response }\end{array}$ \\
\hline$m \mu$ & \\
315 & 0.00 \\
313 & .03 \\
310 & .11 \\
305 & 33 \\
302 & .55 \\
300 & .82 \\
297 & 1.00 \\
295 & .95 \\
290 & .26 \\
289 & .22 \\
285 & .09 \\
280 & .06 \\
275 & .07 \\
270 & .13 \\
265 & .25 \\
260 & .42 \\
258 & .48 \\
254 & .55 \\
248 & .57 \\
245 & .57 \\
240 & .56 \\
\hline
\end{tabular}

\section{CONCLUSION}

The erythemogenic power of ultra-violet radiation depends upon the intensity of the rays and upon the susceptibility of the skin to different wave lengths. But unlike the spectral sensibility curve of the eye which is perceptibly different for different persons, from the limited data available it appears that the spectral erythemic response curve of the human skin is practically the same for different persons, in spite of the fact that the total energy required to produce an erythema is markedly different.

One difficulty in devising a suitable dosage meter is owing to the fact that a long overexposure to radiation at $254 \mathrm{~m} \mu$ produces only a superficial burn, whereas a slight overexposure to the more deeply penetrating radiation of wave length $313 \mathrm{~m} \mu$ produces a painful blister. For example, in one case it was found that an exposure to the wave length $313 \mathrm{~m} \mu$ for 14 minutes produced a barely perceptible erythema (estimated exposure 15 minutes); 16 minutes was somewhat longer than required for a minimum perceptible erythema; and an exposure of 18 minutes produced a painful blister.

On the basis of the limited number of subjects tested in this work, the total energy required to produce a minimum perceptible erythema upon $1 \mathrm{~cm}^{2}$ of untanned skin, using homogeneous radiation of wave length $297 \mathrm{~m} \mu$ (the wave length of maximum of erythemic susceptibility) in the winter time, when the humidity is low and skin is dry, is of the order of 500,000 ergs. The actual values for the three observers were, respectively, 452, 516, and 645 kiloergs per $\mathrm{cm}^{2}$. 
During the summer time (high humidity, skin moist) the values, for these three observers, were respectively 221, 309, and 190 kiloergs per $\mathrm{cm}^{2}$, or an average of about 240 kiloergs. In other words, the average time of exposure for producing a minimum perceptible erythema was reduced to one-half the winter value. The most conspicuous change was on J. M. H., whose exposure time was reduced to one-third the winter value.

When one considers the ever-increasing number of ultra-violet radiators sold for healing purposes, in some of which about 97 per cent of the total erythemogenic radiation, of wave lengths 200 to $315 \mathrm{~m} \mu$ is contained in the highly germicidal but nonpenetrating rays at $254 \mathrm{~m} \mu$ (the resonance line in a so-called "cold" quartz mercury lamp), while other lamps contain practically no ultra-violet radiation of wave lengths shorter than $300 \mathrm{~m} \mu$, but emit longer wave lengths that are more penetrating and more potent in causing burns, the urgent need of a means of standardization of the dosage seems apparent.

In a subsequent paper data will be presented correlating the radiometric "dosage" measurements and the erythemal reaction of the standard sources of ultra-violet radiation now available for therapeutic purposes, as outlined in a preceding paper (1) in which a practical application was made of the herein-described data.

\section{BIBLIOGRAPHY}

1. W. W. Coblentz, R. Stair, and J. M. Hogue, A Balanced Thermocouple and Filter Method of Ultra-violet Radiometry with Practical Applications, B. S. Jour. Res., vol. 7 (RP370), p. 723, 1931.

2. W. W. Coblentz, R. Stair, and J. M. Hogue, The Spectral Erythemic Reaction of the Human Skin to Ultra-violet Radiation, Proc. Nat. Acad. Sci., vol. 17, p. $401,1931$.

3. Hausser and Vahle, Strahelentherapie, vol. 13, p. 41, 1921; vol. 28, p. 25, 1928.

4. Luckiesh, Holladay, and Taylor, J. Opt. Soc. Am., vol. 20, p. 423, 1930.

5. Coblentz and Kahler, B. S. Sci. Papers, vol. 16 (No. 378), p. 233, 1920.

6. Coblentz, B. S. Bull., vol. 11 (No. 227), p. 87, 1914.

7. Coblentz, B. S. Bull., vol. 11 (No. 229), p. 131, 1914.

8. Coblentz, J. Frank. Inst., vol. 175, p. 151, 1913.

9. Luckiesh, M., "Artificial Sunlight," p. 77, 1930; Trans. Illum. Eng. Soc., vol. 26, p. 706, 1931.

Washington, February 2, 1932. 